\title{
Mixed ultrasoft/norm-conserved pseudopotential scheme
}

\section{Stokbro, Kurt}

\section{Published in:}

Physical Review B

Link to article, DOI:

10.1103/PhysRevB.53.6869

Publication date:

1996

\section{Document Version}

Publisher's PDF, also known as Version of record

Link back to DTU Orbit

Citation (APA):

Stokbro, K. (1996). Mixed ultrasoft/norm-conserved pseudopotential scheme. Physical Review B, 53(11), 68696872. https://doi.org/10.1103/PhysRevB.53.6869

\section{General rights}

Copyright and moral rights for the publications made accessible in the public portal are retained by the authors and/or other copyright owners and it is a condition of accessing publications that users recognise and abide by the legal requirements associated with these rights.

- Users may download and print one copy of any publication from the public portal for the purpose of private study or research.

- You may not further distribute the material or use it for any profit-making activity or commercial gain

- You may freely distribute the URL identifying the publication in the public portal

If you believe that this document breaches copyright please contact us providing details, and we will remove access to the work immediately and investigate your claim 


\title{
Mixed ultrasoft/norm-conserved pseudopotential scheme
}

\author{
K. Stokbro \\ Scuola Internazionale Superiore di Studi Avanzati, via Beirut 4, I-34014 Trieste, Italy \\ and Mikroelektronik Centret, Danmarks Tekniske Universitet, Bygning 345b, DK-2800 Lyngby, Denmark
}

(Received 12 October 1995)

\begin{abstract}
A variant of the Vanderbilt ultrasoft pseudopotential scheme, where the norm conservation is released for only one or a few angular channels, is presented. Within this scheme some difficulties of the truly ultrasoft pseudopotentials are overcome without sacrificing the pseudopotential softness. (i) Ghost states are easily avoided without including semicore shells. (ii) The ultrasoft pseudo-charge-augmentation functions can be made softer. (iii) The number of nonlocal operators is reduced. The scheme will be most useful for transition metals, and the feasibility and accuracy of the scheme is demonstrated for the $4 d$ transition-metal rhodium.
\end{abstract}

With the development of the Vanderbilt ultrasoftpseudopotential (US) technique ${ }^{1}$ it has become possible to describe traditionally hard pseudopotential elements like transition metals and first row elements with a modest planewave cutoff. However, for some elements it is difficult to avoid the appearance of so-called ghost states without including semicore states in the pseudopotential construction. ${ }^{2}$ Furthermore, sometimes it is necessary to describe the US pseudoaugmentation charge with a higher cutoff than the pseudocharge of the wave-function sum, and therefore socalled double-grid techniques have been developed. ${ }^{3}$ Both of these aspects significantly reduce the computational efficiency of the US scheme, and it is desirable to find a simple scheme to avoid these difficulties.

In this report I will present a variant of the US technique, where only the normconservation (NC) is released for some of the angular channels. The benefit from this construction is that the semilocal potential of one of the chemical active channels may be taken as the local potential. This reduces the number of projectors in the US scheme and by choosing the semilocal potential of the least bound reference state as the local potential, the appearance of ghost states is effectively avoided. Furthermore, since with this construction there are less pseudocharge-augmentation functions, I have found the pseudocharge smoothening procedure of Ref. 3 to be more efficient. In the following I will briefly review the main features of the US scheme, and present the modifications in a mixed US/NC scheme. Finally, I will build a US/NC pseudopotential for Rh and compare the transferability and computational efficiency with that of a NC pseudopotential and an US pseudopotential with semicore shells.

In the US scheme the pseudo-wave-functions do not obey a NC condition and it is this feature that makes it possible to construct pseudopotentials with a modest plane-wave cutoff $(\leqslant 30 \mathrm{Ry})$ for traditionally hard pseudopotential elements. To make the pseudopotential retain the first-order scattering properties of the all-electron potential, as is the case of the NC pseudopotentials, the pseudo-wave-functions have to obey a generalized eigenvalue equation, where the missing norm enters in the overlap matrix. Furthermore, the density cannot simply be constructed from the pseudo-wavefunctions, an augmentation charge has to be added in order to retain the correct electrostatic potential in the interstitial region. Given a set of occupied pseudo-wave-functions $\left\{\Phi_{\alpha}\right\}$, the augmentation charge is given by

$$
\begin{gathered}
\rho^{\mathrm{aug}}(\mathbf{r})=\sum_{\alpha, n, m}\left\langle\Phi_{\alpha} \mid \chi_{n}\right\rangle Q_{n m}(\mathbf{r})\left\langle\chi_{m} \mid \Phi_{\alpha}\right\rangle, \\
Q_{n m}(\mathbf{r})=\psi_{n}^{*}(\mathbf{r}) \psi_{m}(\mathbf{r})-\phi_{n}^{*}(\mathbf{r}) \phi_{m}(\mathbf{r}),
\end{gathered}
$$

where $\psi_{n}$ are the reference all-electron atomic wave functions, $\phi_{n}$ are the corresponding reference atomic pseudowave-functions, and $\chi_{m}$ are projectors upon the reference atomic pseudo-wave-functions.

With this choice for the augmentation charge the total pseudodensity not only has the same norm as the all-electron density, as in the case of NC pseudopotentials, but is identical to the all-electron density in the case where the set of reference states inside each core forms a complete basis for the all-electron wave functions and the pseudo-wave functions, i.e., $\Psi_{\alpha}=\Sigma_{n} a_{n}^{\alpha} \psi_{n}$ and $\Phi_{\alpha}=\Sigma_{n} b_{n}^{\alpha} \phi_{n}$. To see this, observe that since the pseudo- and all-electron wave functions and their radial derivatives coincide at the core radius they must have the same expansion in the reference states, thus $a_{n}^{\alpha}=b_{n}^{\alpha}$, which implies that $\Sigma_{\alpha}\left|\Psi_{\alpha}\right|^{2}=\Sigma_{\alpha}\left|\Phi_{\alpha}\right|^{2}+\rho^{\text {aug }}$.

Therefore, in the US scheme not only is the electrostatic monopole of the core region correct, but also higher-order poles are described rather accurately. Another feature of the US scheme is the possibility of including several reference states for each angular channel.

In the NC scheme it is common practice to take one of the semilocal potentials as the local potential and thereby reduce the number of semilocal channels that have to be described. Furthermore, by choosing the semilocal potential of the least bound reference state one can effectively avoid the appearance of ghost states ${ }^{4,5}$ in a Kleinmann-Bylander ${ }^{6}$ implementation. A similar choice for the local potential in the ultrasoft scheme would produce a very poor pseudopotential, since due to the missing norm of the corresponding pseudo-wavefunction the local potential would only have the zero-order scattering properties of the all-electron potential. Instead the common practice is to construct a semilocal potential for the first angular channel that is not chemical active and use that for the local potential; for instance, for the transition metals the semilocal potential of the $f$ channel is usually used for 

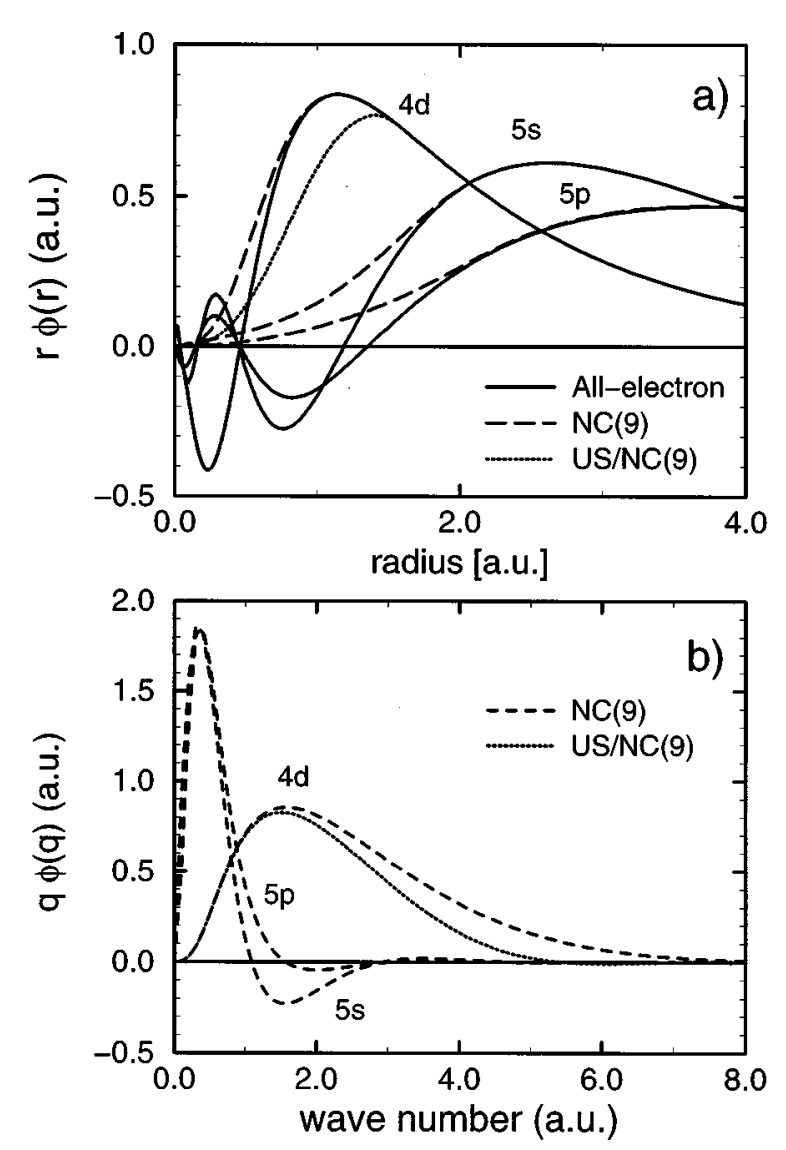

FIG. 1. (a) The real-space all-electron wave functions of Rh and the pseudo-wave-functions of the NC(9) and US/NC(9) pseudopotentials. (b) The Fourier-transformed pseudo-wave-functions.

the local potential. However, for most transition metals this will produce a local potential with bound states far below the $s$ - and $p$-reference eigenstates, and the appearance of ghost states is therefore unavoidable. ${ }^{5}$ A solution to this problem is to include $s$ - and $p$-semicore shells in the pseudopotential construction; since these new reference eigenstates usually are below the bound states of the local potential the ghost states will disappear. The inclusion of semicore shells has the benefit that the accuracy of the pseudopotential becomes comparable to all-electron calculations; ${ }^{7}$ however, it also increases the computer time and memory requirements of the calculation substantially.

As an alternative I propose a mixed US/NC scheme, where the normconservation is only released for the hard channels, and the semilocal potential of one of the soft channels may thereby be taken as the local potential. For most transition metals the normconservation will only have to be released for the $d$ channel, and the semilocal potential of the $p$ channel can therefore be taken as the local potential, thereby avoiding the appearance of ghost states. The pseudocharge-augmentation functions $Q_{n m}$ of Eq. (2) I now define as

$$
Q_{n m}(\mathbf{r})=\xi_{n}^{*}(\mathbf{r}) \xi_{m}(\mathbf{r})-\phi_{n}^{*}(\mathbf{r}) \phi_{m}(\mathbf{r})
$$

where $\xi=\psi$ for the US channels and $\xi=\phi$ for the NC channels, and only contributions from nonlocal channels are in-

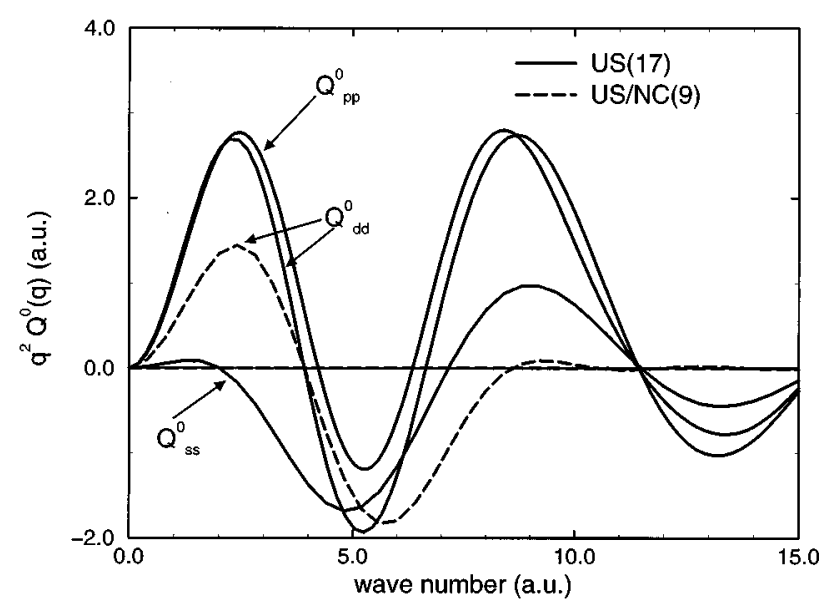

FIG. 2. The Fourier-transformed pseudo-charge-augmentation functions of the US(17) and the US/NC(9) pseudopotentials.

cluded; i.e., for most transition metals only the $Q_{d d}$ and $Q_{s d}$ elements will contribute to the augmentation charge.

In the following I will construct a scalar-relativistic mixed US/NC pseudopotential for the $4 d$ transition metal $\mathrm{Rh}$, show that the error introduced by the choice for the augmentation charge is minute, and compare the transferability and computational efficiency with a NC pseudopotential and a US pseudopotential with semicore states.

To construct the NC pseudopotential [denoted NC(9)] I used the procedure suggested by Troullier and Martins, ${ }^{8}$ with core radii $2.53,2.53$, and 1.39 (a.u.) for the $s, p$, and $d$ channels, respectively. As suggested in Ref. 9, only the semilocal $s$ and $d$ potentials were constructed in the atomic ground-state configuration $4 d^{8} 5 s^{1}$, while the semilocal $p$ potential was constructed in the $4 d^{7} 5 s^{0.75} p^{0.25}$ configuration. Figure 1 shows the pseudo-wave-functions and their Fourier transforms. From the latter it can be seen that the $s$ and $p$ wave functions are converged at $\approx 20-25 \mathrm{Ry}$, while the $d$ wave function must be described with a cutoff of $\approx 50-60$ Ry. From this pseudopotential the mixed US/NC pseudopotential [denoted US/NC(9)] was constructed by releasing the norm of the $d$ wave function, increasing its core radius to 1.6 a.u, and then using the smoothening procedure of Refs. 10 and 7 to generate $d$ ultrasoft pseudo-wave-functions for two reference states, where one has the atomic eigenvalue $(-0.4518 \mathrm{Ry})$ and the other the energy (0.2 Ry). With this construction the $d$ pseudo-wave-function can be described with a cutoff of $\approx 25-30 \mathrm{Ry}$, as seen from its Fourier transform shown in Fig. 1(b) (dotted line). Finally, I have constructed an US pseudopotential including $4 s$ and $4 p$ semicore shells [denoted US(17)], using two reference states for each channel, and a core radius of 2.0, 2.0, and 1.6 for the $s$, $p$, and $d$ channel, respectively. The US(17) pseudopotential is also converged at $\approx 25-30$ Ry.

To generate pseudo-charge-augmentation functions I have used the procedure described in Refs. 3 and 7, where the augmentation functions are replaced by $L$-dependent counterparts $Q_{n m}^{L}$,

$$
Q_{n m}(\mathbf{r})=\sum_{L M} c_{L M}^{n m} Y_{L M}(\hat{r}) Q_{n m}^{L}(r)
$$


TABLE I. Comparison of the three rhodium pseudopotentials. US(17) is the ultrasoft pseudopotential including semicore shells, $\mathrm{NC}(9)$ is the normconserved pseudopotential, and US/NC(9) is the mixed normconserved/ultrasoft scheme. In all the calculations, including the atomic calculations of (a), the pseudopotentials are used in a nonlocal separable form. (a) The calculated atomic-eigenvalues in different reference configurations and their deviation from the all-electron values. (b) The lattice constant $\left(a_{0}\right)$, bulk modulus $(B)$, derivative of the bulk modulus $\left(B^{\prime}\right)$, and cohesive energy of rhodium $\left(E_{\mathrm{coh}}\right)$. (c) The surface energy $\left(E_{\text {surf }}\right)$, work function $(W)$, and interlayer relaxation $\left(\Delta_{12}, \Delta_{23}\right)$ of the $\operatorname{Rh}(110)$ surface. The US(17)* pseudopotential neglects pseudo-charge-augmentation functions that couple different angular channels, i.e., $Q_{s p}=Q_{p d}=Q_{s d}=0$.

\begin{tabular}{|c|c|c|c|c|c|c|c|}
\hline \multicolumn{8}{|c|}{ (a) Atomic properties of $\mathrm{Rh}$} \\
\hline Pseudopotential & Configuration & $\begin{array}{c}4 d \\
(\mathrm{Ry})\end{array}$ & $\begin{array}{l}\Delta(4 d) \\
(\mathrm{mRy})\end{array}$ & $\begin{array}{c}5 s \\
(\mathrm{Ry})\end{array}$ & $\begin{array}{l}\Delta(5 s) \\
(\mathrm{mRy})\end{array}$ & $\begin{array}{l}5 p \\
\text { (Ry) }\end{array}$ & $\begin{array}{l}\Delta(5 p) \\
(\mathrm{mRy})\end{array}$ \\
\hline $\mathrm{US}(17)$ & $4 d^{9} 5 s^{0} 5 p^{0}$ & -0.28267 & 0.0 & -0.26864 & 0.0 & -0.03501 & 0.0 \\
\hline US/NC(9) & $4 d^{9} 5 s^{0} 5 p^{0}$ & -0.28155 & 1.2 & -0.27227 & -3.6 & -0.03798 & -3.0 \\
\hline $\mathrm{NC}(9)$ & $4 d^{9} 5 s^{0} 5 p^{0}$ & -0.27479 & 7.9 & -0.27022 & -1.6 & -0.03883 & -3.8 \\
\hline $\mathrm{US}(17)$ & $4 d^{7} 5 s^{1} 5 p^{0}$ & -1.24280 & 0.1 & -0.91783 & 0.0 & -0.53999 & 0.1 \\
\hline US/NC(9) & $4 d^{7} 5 s^{1} 5 p^{0}$ & -1.24384 & -0.9 & -0.91472 & 3.1 & -0.53760 & 2.5 \\
\hline $\mathrm{NC}(9)$ & $4 d^{7} 5 s^{1} 5 p^{0}$ & -1.25787 & -15.0 & -0.91985 & -2.0 & -0.53800 & 2.1 \\
\hline \multicolumn{8}{|c|}{ (b) Bulk properties of Rh } \\
\hline Pseudopotential & $\begin{array}{c}\text { Cutoff } \\
\text { (Ry) }\end{array}$ & $\begin{array}{l}a_{0} \\
(\AA)\end{array}$ & $\begin{array}{c}B \\
\text { (Mbar) }\end{array}$ & $B^{\prime}$ & $\begin{array}{c}E_{\text {coh }} \\
\text { (Ry/atom) }\end{array}$ & & \\
\hline Expt. & & $3.80^{\mathrm{a}}$ & $2.76^{\mathrm{a}}$ & & $0.42^{\mathrm{a}}$ & & \\
\hline All Electron & & $3.74^{\mathrm{b}}$ & $3.46^{\mathrm{b}}$ & $7.3^{\mathrm{b}}$ & $0.569^{\mathrm{b}}$ & & \\
\hline US(17) & 30 & 3.75 & 3.44 & 7.8 & 0.636 & & \\
\hline US/NC(9) & 30 & 3.81 & 3.16 & 5.6 & 0.609 & & \\
\hline $\mathrm{NC}(9)$ & 60 & 3.86 & 2.98 & 5.5 & 0.594 & & \\
\hline \multicolumn{8}{|c|}{ (c) Surface properties of $\mathrm{Rh}(110)$} \\
\hline Pseudopotential & $\begin{array}{c}\text { Cutoff } \\
\text { (Ry) }\end{array}$ & $\begin{array}{l}\text { CPU time } \\
\text { (s) }\end{array}$ & $\begin{array}{c}\mathrm{E}_{\text {surf }} \\
(\mathrm{eV} / \text { atom })\end{array}$ & $\begin{array}{c}\mathrm{W} \\
(\mathrm{eV})\end{array}$ & $\begin{array}{c}\Delta d_{12} \\
(\%)\end{array}$ & $\begin{array}{c}\Delta d_{23} \\
(\%)\end{array}$ & \\
\hline Expt. & & & & $4.98^{\mathrm{a}}$ & $-6.9^{c}$ & $1.9^{\mathrm{c}}$ & \\
\hline $\mathrm{US}(17)$ & 30 & 5796 & 1.73 & 4.77 & -12.1 & 4.9 & \\
\hline US/NC(9) & 30 & 1514 & 1.92 & 4.99 & -10.2 & 2.5 & \\
\hline $\mathrm{NC}(9)$ & 60 & 5162 & 1.87 & 4.98 & -10.5 & 2.6 & \\
\hline $\mathrm{US}(17)^{*}$ & 30 & 5796 & 1.73 & 4.78 & -12.1 & 4.9 & \\
\hline
\end{tabular}

${ }^{\mathrm{a}}$ Reference 11 .

${ }^{\mathrm{b}}$ Reference 12 .

${ }^{\mathrm{c}}$ Reference 13 .

which are smoothened inside a core radius $r_{\text {in }}^{L}$, subject to the condition that the $L$ th moment of the electron charge density is conserved. One difficulty with this method is that if the core radius $r_{\text {in }}^{0}$ is extended beyond a certain radius the smoothening procedure starts to develop negative sections in the $Q_{n m}^{0}(r)$ terms, leading to negative pseudo-chargedensities. I have found that this difficulty is related to the different node structure of the radial all-electron wave functions, and that a larger $r_{\text {in }}^{0}$ core radius can be chosen when there is only one angular channel. Figure 2 shows the $Q_{s s}^{0}$, $Q_{p p}^{0}$, and $Q_{d d}^{0}$ pseudo-charge-augmentation functions of the US(17) pseudopotential and the $Q_{d d}^{0}$ pseudo-chargeaugmentation function of the US/NC(9) pseudopotential. For the US(17) pseudopotential the core radius could not be extended beyond $r_{\text {in }}^{0}=0.6$ a.u., while the US/NC(9) core radius was $r_{\text {in }}^{0}=0.9$ a.u. giving softer pseudo-charge-augmentation functions.
Table I shows the transferability of the US/NC(9), NC(9), and US(17) pseudopotentials for atomic, bulk, and surface properties of Rh. First notice the very high quality of the US(17) pseudopotential, having an accuracy essentially identical to all-electron calculations. Notice also the discrepancy (due to the local-density approximation) between the allelectron calculation and the experimental results for the bulk properties of $\mathrm{Rh}$, which warns that the quality of a pseudopotential should never be judged by comparison with experimental data. The atomic calculations show that the main difference between the $\mathrm{NC}(9)$ and the US/NC(9) pseudopotentials, is the latter's enhanced description of the $d$ electrons, which is due to the use of two reference states.

Table I(c) shows the CPU time used to obtain the selfconsistent charge density of the nonrelaxed surface for the three different pseudopotential schemes. The comparison reveals that the US/NC(9) pseudopotential is 3.8 times faster that the US(17) pseudopotential and 3.4 times faster than the 
NC(9) pseudopotential. For large systems the orthogonalization step is the most time-consuming part of a plane-wave program, and this scales as $O\left(N M^{2}\right)$, where $N$ is the number of plane waves and $M$ the number of occupied bands. In the present case this gives a time factor relative to the US/NC(9) pseudopotential of $(17 / 9)^{2} \approx 3.6$ for the US $(17)$ pseudopotential and $2^{3 / 2} \approx 2.8$ for the $\mathrm{NC}(9)$ pseudopotential. Another important issue is the memory requirement that scales as $O(N M)$, which results in a factor $17 / 9 \approx 1.9$ for the US(9) pseudopotential and a factor $2^{3 / 2} \approx 2.8$ for the $\mathrm{NC}(9)$ pseudopotential relative to the US/NC(9) pseudopotential.

To test whether the neglect of the $Q_{p d}$ pseudo-chargeaugmentation functions in the US/NC scheme introduces any significant error, I have constructed a US(17)* pseudopotential with $Q_{s p}=Q_{p d}=Q_{s d}=0$. These terms are most important for asymmetric systems; however, the calculated $\mathrm{Rh}$ (110) surface properties are almost unaffected by this approximation(see Table Ic). Furthermore, I have found that different variations of the US/NC scheme as including two $s$-reference states, using the atomic ground state for the $p$-reference configuration or including the $Q_{s s}$ term, do not change the quality of the pseudopotential significantly. The discrepancy between the US(17) and US/NC(9) pseudopo- tentials is therefore mainly due to the latter's neglect of semicore states.

In conclusion, I have presented a simple scheme for constructing a mixed normconserved/ultrasoft pseudopotential using as a starting point a normconserved pseudopotential. The resulting pseudopotential scheme is more accurate and computationally efficient than the initial normconserved pseudopotential. Compared to truly ultrasoft schemes, ghost states are avoided without including semicore shells and the ultrasoft pseudoaugmentation charge is more easily described with the same cutoff as the wave-function pseudocharge.

I acknowledge D. Vanderbilt for valuable discussion and for providing an atomic program for the US pseudopotential construction, and P. Giannozzi for help with constructing the NC pseudopotential. I also thank V. Fiorentini for the allelectron data for the bulk properties of $\mathrm{Rh}$, and $\mathrm{S}$. Baroni for directing me towards the ultrasoft method and for valuable discussions concerning the method. This work was done under support of the Danish research councils, EEC Contract No. ERBCHBGCT 920180, EEC Contract No. ERBCHRXCT 930342, and CNR project Supaltemp.
${ }^{1}$ D. Vanderbilt, Phys. Rev. B 41, 7892 (1990).

${ }^{2} \mathrm{D}$. Vanderbilt (private communication).

${ }^{3}$ K. Laasonen et al., Phys. Rev. B 47, 10142 (1993).

${ }^{4}$ P. E. Blöchl, Phys. Rev. B 41, 5414 (1990).

${ }^{5}$ X. Gonze, R. Stumpf, and M. Scheffler, Phys. Rev. B 44, 8503 (1991).

${ }^{6}$ L. Kleinman and D. M. Bylander, Phys. Rev. Lett. 48, 1425 (1982).

${ }^{7}$ R. D. King-Smith and D. Vanderbilt, Phys. Rev. B 49, 5828 (1994).

${ }^{8}$ N. Troullier and J. L. Martins, Phys. Rev. B 43, 1993 (1991).
${ }^{9}$ G. B. Bachelet, D. R. Hamann, and M. Schlüter, Phys. Rev. B 26, 4199 (1982).

${ }^{10}$ A. M. Rappe, K. M. Rabe, E. Kaxiras, and J. D. Joannopoulos, Phys. Rev. B 41, 1227 (1990).

${ }^{11}$ CRC Handbook of Chemistry and Physics, 75th ed., edited by D. R. Lide (CRC Press, Boca Raton, 1994).

${ }^{12} \mathrm{~V}$. Fiorentini (private communication). The calculations were performed with the full-potential linear-muffin-tin-orbital (LMTO) method, treating the $4 s, 4 p, 4 d, 5 s$, and $5 p$ states as valence states, and relaxing the core states.

${ }^{13}$ W. Nichtl et al., Surf. Sci. 188, L729 (1987). 